Article

\title{
Design, Measurement and Shape Reconstruction of Soft Surgical Actuator Based on Fiber Bragg Gratings
}

\author{
Yanlin He ${ }^{1,2}$, Lianqing Zhu ${ }^{1,2, *}$, Guangkai Sun ${ }^{1,2}$, Mingxin Yu ${ }^{1,2}$ and Mingli Dong ${ }^{1,2, *}$ \\ 1 Beijing Engineering Research Center of Optoelectronic Information and Instruments, \\ Beijing Information Science and Technology University, Beijing 100192, China; \\ heyanlin@bit.edu.cn (Y.H.); Guangkai.sun@buaa.edu.cn (G.S.); mingxinbit@gmail.com (M.Y.) \\ 2 Bionic and Intelligent Equipment Lab, Beijing Information Science and Technology University, \\ Beijing 100192, China \\ * Correspondence: lianqingzhu18@gmail.com (L.Z.); dongml@bistu.edu.cn (M.D.); \\ Tel.: +86-010-8417-6477 (L.Z.)
}

Received: 19 August 2018; Accepted: 20 September 2018; Published: 30 September 2018

\begin{abstract}
Soft actuators are the components responsible for organs and tissues adsorptive fixation in some surgical operations, but the lack of shape sensing and monitoring of a soft actuator greatly limits their application potential. Consequently, this paper proposes a real-time 3D shape reconstruction method of soft surgical actuator which has an embedded optical fiber with two Fiber Bragg Grating (FBG) sensors. First, the design principle and the sensing of the soft actuator based on FBG sensors are analyzed, and the fabrication process of soft actuator which has an embedded optical fiber with two FBG sensors is described. Next, the calibration of the FBG sensors is conducted. Based on curvatures and curve fitting functions, the strategy of 3D shapes reconstruction of the soft actuator is presented. Finally, some bending experiments of the soft actuator are carried out, and the 3D shapes of the soft actuator at different bending states are reconstructed. This well reconstructed 3D shape of a soft actuator demonstrates the effectiveness of the shape reconstruction method that is proposed in this paper, as well as the potential and increased applications of these structures for real soft surgical actuators.
\end{abstract}

Keywords: micro fiber sensor; shape reconstruction; soft surgical robot; pneumatic actuator; modeling

\section{Introduction}

In recent years, soft actuators are widely used to implement surgical tasks due to its large-scale deformation and compliant adaptation to meet the complex elastic structures of the human organs and tissues [1-3]. The soft actuators can deform to absorb the energy generated by a variety of stimulation, including electrical charges, hydraulic actuating, pneumatic actuating and chemical reactions, etc. In particular, considering the advantages of easy fabrication, low material cost and lightweight, etc., the pneumatic actuating soft actuators are promising for surgical applications [4], and the use of soft manipulators in surgical operations could overcome the limitations of the current rigid surgical system. Consequently, compared with some traditional rigid and hard continuum surgical manipulators, the soft biological materials could considerably reduce the harm, which is caused by the robotic manipulators, and the soft surgical manipulators are inherently compliant and intrinsically safe in the interaction with humans.

While current researchers have emphasized the promising potential of soft manipulators in surgical applications, the lack of complicate shape measurement and monitoring for soft actuator are greatly limiting their practical application. In order to reliably and widely use these actuators in soft surgical manipulator, mastering the relationship between shape measurement and position 
control of the actuator is indispensable. Considering the flexible bodies of the soft manipulator, some conventional rigid sensors, like encoders and strain gauges $[5,6]$ have problems of complex cable networks, electromagnetic and noise signal interference, etc., and the rigid sensor will damage human tissue when the manipulator interacts with the human organs surround it [7-9]. The visual-based sensing systems are also inapplicable due to the narrow spaces and various obstacles in human body. At present, the embedded flexible sensing devices and methods that can be applied to the shape measurement and position control of soft surgical actuators are desired.

Different from above-mentioned sensors, the optical fiber sensors have advantages of small size, light weight, fast response, small transmission loss, not be affected with electromagnetic interference and easy to be buried into the interior of the material $[10,11]$. More importantly, the optical fiber sensor is harmless when in contact with human tissues and organs [12-15], and the optical fiber sensor can be embedded into the flexible body of the soft manipulator to realize its morphological sensing. Consequently, these distinctive characteristics of the optical fiber sensor make it more advantageous, and the prospects for soft surgical robotics and biomedical applications; and several types of research of optical fiber sensors have been investigated in recent years. In [12], the fiber sensors were embedded into a piece of garment, and the motion performance of the patient was evaluated by the angle of joint, which is monitored by the embedded fiber sensors; this sensing garment is comfortable for patients and has good response to the flexion motion of the elbow. As proposed in [13], the bending state of a manipulator was measured by an optical fiber sensing system and the mechanism of this sensing system based on a laser power modulation. Although this sensing system has good accuracy in measuring bending angles, bending radius and orientation of the flexible segment, its 3D shape reconstruction and visualization monitoring are not realized. In addition, this sensing system adopts three optical fibers to interpolate the bending radius and the elongation of the manipulator, and this undoubtedly increases the complexity of the theoretical models of the manipulator. In [12], some micro-optical sensors were integrated into soft robot arms to form their pose sensing system; this system couples the optical fibers with some passive cables mechanically in a basal unit that has sensing function, and a distance modulation array is integrated with the basal sensing unit. The pose of the soft robot arms can be calculated by the mechanical deformation to the voltage variation of the sensing signal. This sensing system can be used to measure the length and bending angle of soft robot arms, their 3D shape reconstruction and monitoring are not attained. Zhao et al. [15] designed a hardware and control method for a soft orthosis, the initial quantification of its force augmenting capabilities was performed, and its closed-loop control was implemented through the feedback from the optical-fiber sensors which was embedded into the actuator.

However, few studies have been conducted in real-time; 3D shape reconstruction and visualization monitoring of soft surgical actuator which are embedded a in single optical fiber with Fiber Bragg Grating (FBG) sensors. In this paper, to understand real-time shape monitoring of the soft actuator, a 3D shape reconstruction method based on two FBG sensors is proposed. First, an optical fiber with two FBG sensors is embedded into the soft actuator; then the calibration of FBG sensors is conducted, the spectrum and wavelength shift caused by the bending of the soft actuator is also measured; and finally, the 3D shape of the soft actuator is reconstructed based on the interpolation and curve fitting functions.

The remainder of this paper is organized as follows. In Section 2, we describe the mechanical design and modeling of the soft silicone actuator. In Section 3, we introduce the sensing theory and shape measurement method of FBG sensors. The experimental setup and testing of the soft actuator are elaborate in Section 4. Section 5 concludes the paper. 


\section{Design and Modeling of Soft Actuator with Embedded FBG Sensors}

\subsection{Structure Design and Analytical Modeling}

Through adjusting some dimension parameters of the soft actuator, including its length, the diameter of the hemi-circle chamber, the pneumatic chamber's wall thickness, the fiber-winding angle and orientation, etc., The design details of the soft actuator are presents in Figure 1 [16].

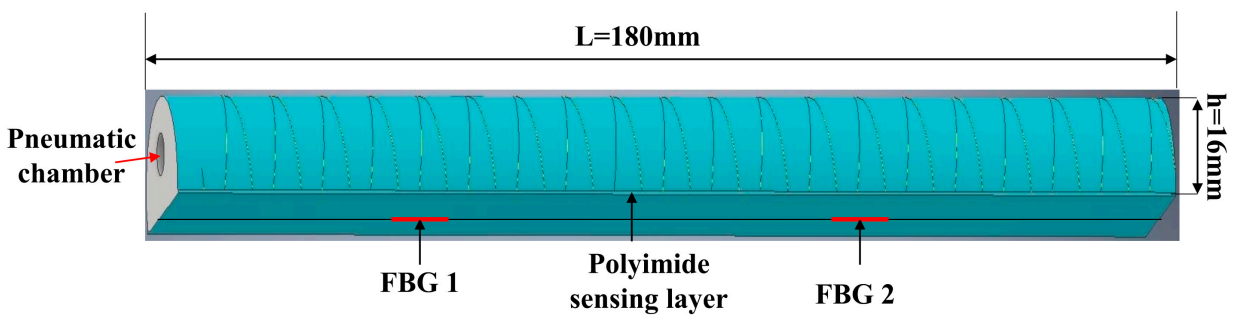

(a)

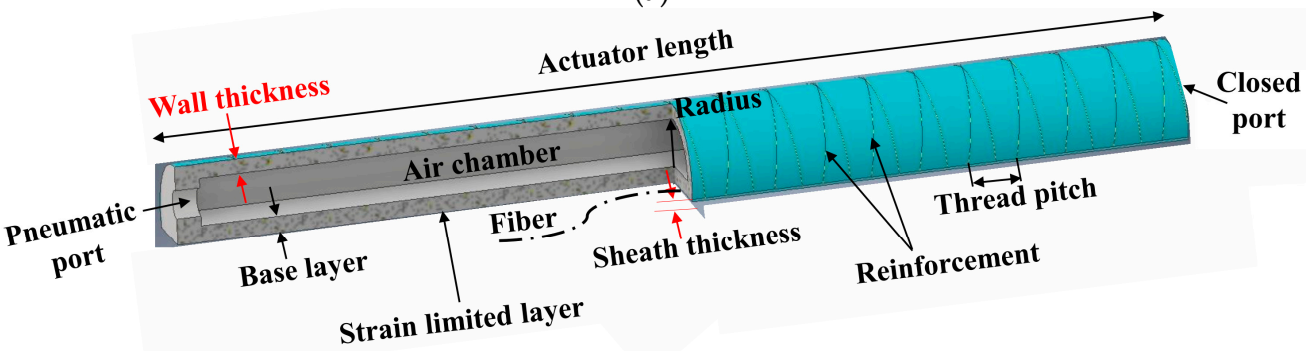

(b)

Figure 1. Design of the proposed Fiber Bragg Grating (FBG) soft pneumatic actuator. (a) FBG-based polyimide sensing layer and its location. (b) Geometrical parameters and design variables of soft fiber-reinforced actuator.

The dimensions of the hemi-circular shape are achieved (see Figure 2a) using the cross-sectional area of $\pi a^{2} / 2$, and the wall thickness of the actuator is $t=a / 4$. With an input air pressure of $P_{i n}$, the bending torques $\left(M_{a}\right)$ of internal air pressure relative to the closed port of the soft actuator are expressed as $[17,18]$ :

$$
M_{a}^{H C}=0.34 a^{3} P_{\text {in }}
$$

Considering the characteristics of the hyper-elastic material of silicone and the dimension of the soft actuator; the relationship between the input pressure and the bending angle were analyzed. The variables in the model were actual actuator dimensions and material properties, that could be either measured or obtained from calibrations. From the model of incompressible Neo-Hookean $(\mathrm{NH})$ material [4], the strain energy of the soft actuator can be written as:

$$
W=\frac{\mu}{2}\left(I_{1}-3\right)=\frac{\mu}{2}\left(\lambda_{1}^{2}+\lambda_{2}^{2}+\lambda_{3}^{2}-3\right)
$$

where $\mu$ represents the initial shear modulus of the silicone, $I_{1}$ represents the first constant of axial, circumferential and radial principal. $\lambda_{1}$ represents the primary stretch in the axial direction of the actuator, $\lambda_{2}(=1)$ represents the circumferential strain, and it was ignored due to the fiber reinforcement constraint, and $\lambda_{1} \lambda_{2} \lambda_{3}=1$ due to the incompressibility of the material, then it was obtained that:

$$
\lambda_{1}=\lambda, \quad \lambda_{2}=1, \quad \lambda_{3}=\frac{1}{\lambda}
$$

When supplying the pressure $\left(P_{1}>P_{a t m}\right)$ into the air chamber, the top wall of the actuator extended and the non-retractable layer restricted its bottom layer, the actuator bent with a radius $R$ and angle $\theta$ toward the bottom layer (see Figure 2). In addition, the fiber-reinforced structure was regarded as a 
rigid constraint to the soft actuator, and the actuator was simplified as a homogeneous incompressible $\mathrm{NH}$ material [4] whose initial shear modulus is $\bar{\mu}$. In the model of the actuator, some dynamics pressurization effects were ignored, and the actuator had an identical bending curvature.

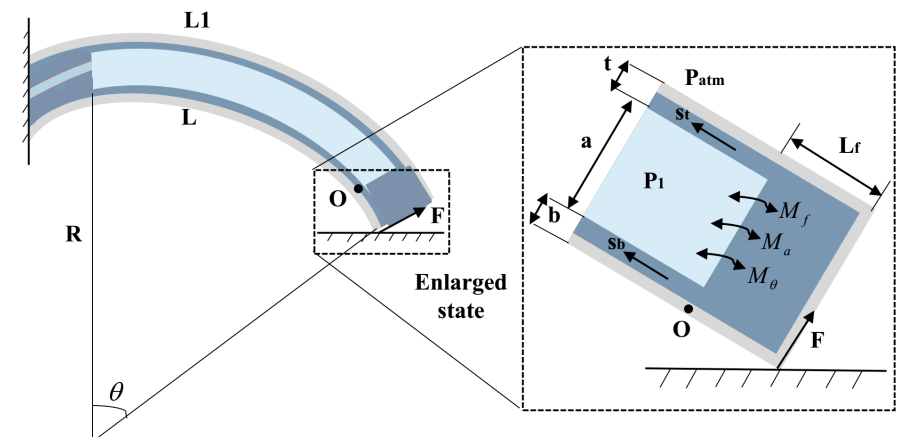

(a)

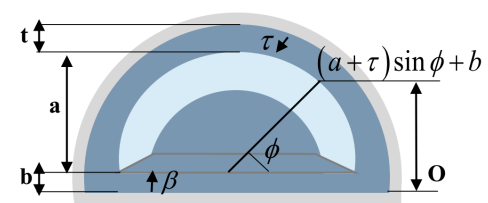

(b)

Figure 2. Different view of the soft actuator and its distal tip in bending state. (a) Side view. (b) Cross-sectional view.

The nominal main strain $s_{i}$ can be defined by $W, \lambda i$. Where $p$, the Lagrange multiplier is:

$$
\begin{gathered}
s_{1}=\frac{\partial W}{\partial \lambda_{1}}-\frac{p}{\lambda_{1}}=\bar{\mu}\left(\lambda-\frac{1}{\lambda^{3}}\right) \\
s_{2}=\frac{\partial W}{\partial \lambda_{2}}-\frac{p}{\lambda_{2}}=\bar{\mu}\left(1-\frac{1}{\lambda^{2}}\right) \\
s_{3}=\frac{\partial W}{\partial \lambda_{3}}-\frac{p}{\lambda_{3}}=0
\end{gathered}
$$

Within the range of stretches considered in soft actuator application $(1 \leq \lambda<1.5)$, the axial stretch $s_{1}$ this paper only considered was denoted as $s$.

The bending moment was induced by the internal strain of the soft actuator, the bending torque and the combined moment at each bending state can be expressed as:

$$
\begin{aligned}
& M_{a}=2\left(P_{1}-P_{a t m}\right) \int_{0}^{\frac{\pi}{2}}(a \sin v+b) a^{2} \cos ^{2} v d v=\frac{4 a^{3}+3 \pi a^{2} b}{6}\left(P_{1}-P_{a t m}\right) \\
& M_{\theta}=\int_{0}^{b} 2 s_{\beta}(a+t) L \beta d \beta+2 \int_{0}^{t} \int_{0}^{\frac{\pi}{2}} s_{\tau, \phi}\left((a+\tau)^{2} \sin \phi+b(a+\tau) L d \phi\right) d \tau
\end{aligned}
$$

where $M_{a}$ is the strain of the soft actuator, $M_{\theta}$ is the combined moment of the strain.

Considering the bending moment produced by the internal strain of the soft actuator, the bending torque $M_{a}$ equal to the combined moment of the strain $M_{\theta}$, and the relationship between input pressure $\left(P_{i n}\right)$ and the actuator's bending angle $(\theta)$ can be written as:

$$
P_{\text {in }}=P_{1}-P_{a t m}=\frac{6 M_{\theta}(\theta)}{4 a^{3}+3 \pi a^{2} b}
$$

where $a$ is the radius of the actuator's air chamber, $b$ is the bottom thickness of the actuator. 


\subsection{The Fabrication and Prototype of the Soft Actuator}

In this section, we describe our procedure for molding the soft actuator The design of the FBG-based optical fiber is embedded into the soft actuator through a polyimide tape, which acted as the strain limited layer; the bending behavior and deformed shape measurement of the soft actuator was realized by the FBG sensor. The soft actuator mainly includes five parts: A hemi-circle elastomeric air chamber which including the caps at the distal and proximal ends; an optical fiber with two FBG sensors is glued on the bottom surface of the silicone layer; some fiber windings are wrapped around the chamber of the actuator; and the whole actuator is encapsulated by a soft silicone sheath [11]. The radial expansion was limited and the linear extension was promoted by the reinforcement of the circumferential fibers, the linear elongation of the bottom surface of the soft actuator was restricted by the strain limited layer, so the part of the soft actuator extends while the strain limited layer restricts expansion along one surface, and thus generating a bending motion as the pressure was inputted into the soft actuator.

The fabrication of a soft actuator with embedded FBG sensors is performed through several steps. First, equal amount of Ecoflex-0050 A and B are mixed and poured into the mold \#1 to fabricate the half round soft actuator with a thickness of $4 \mathrm{~mm}$, and the silicone actuator is cured for 4 hours under indoor temperature. An Objet Connex 500 printer (Objet500 Connex3, Stratasys, Eden Prairie, MN, USA, 2016) is used to printing the two $(\# 1, \# 2)$ molds of the soft actuator, as depicts in Figure 3. Second, the optical fiber with two FBG sensors is put on the bottom surface of the silicone layer, then a polyimide tape is glued on the bottom surface, and thus the optical fiber is fixed to the bottom of the silicone layer. Third, a single Kevlar fiber is used as fiber reinforcement and wrapped around the actuator body in a double helix pattern, and the actuator body is then encapsulated by a $1.0 \mathrm{~mm}$ thick silicone layer putting its entire assembly into the mold \#2. Finally, the mold (\#2) and the half steel rod are extracted from the soft actuator, then some additional silicone is poured on the top and bottom of the actuator in order to enclose the chamber and connect the pipe of pneumatic actuation.

The prototype of the soft actuator with different views are presents in Figure 4, as we can see from Figure 4, an optical fiber with two FBG sensors are embedded into the soft actuator through a polyimide tape, and the optical fiber with FBG sensors are well protected by the polyimide thin film layer. Thus, the FBG-based polyimide sensing layer is formed, and it is embedded into the soft actuator as the strain restricted layer, which served as a primary part to realize bending and shape measurement, and the FBG sensors are stretched with the bending motion of the soft actuator.

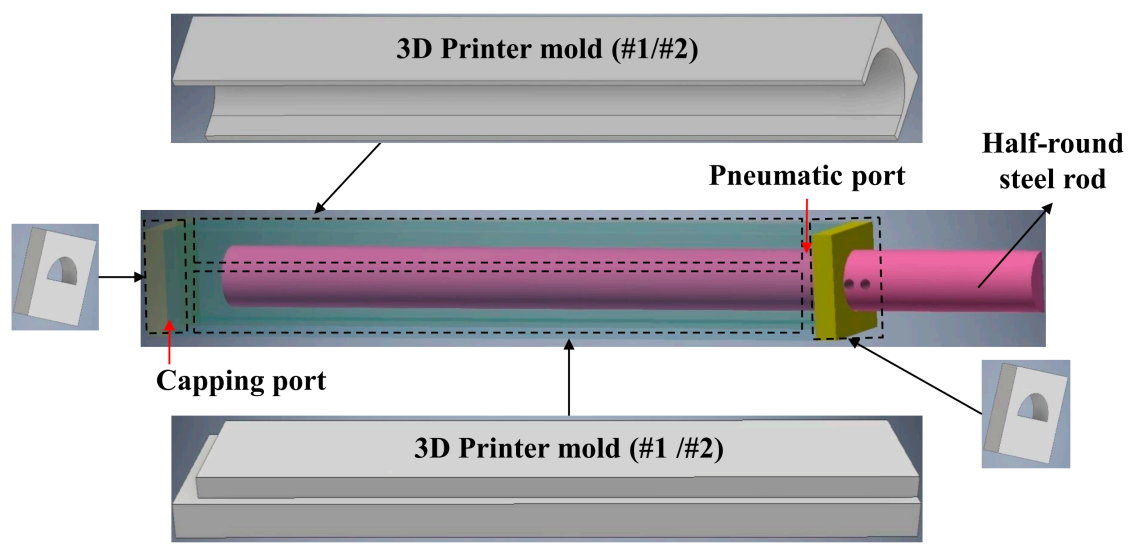

Figure 3. 3D printed mold of the soft actuator. 


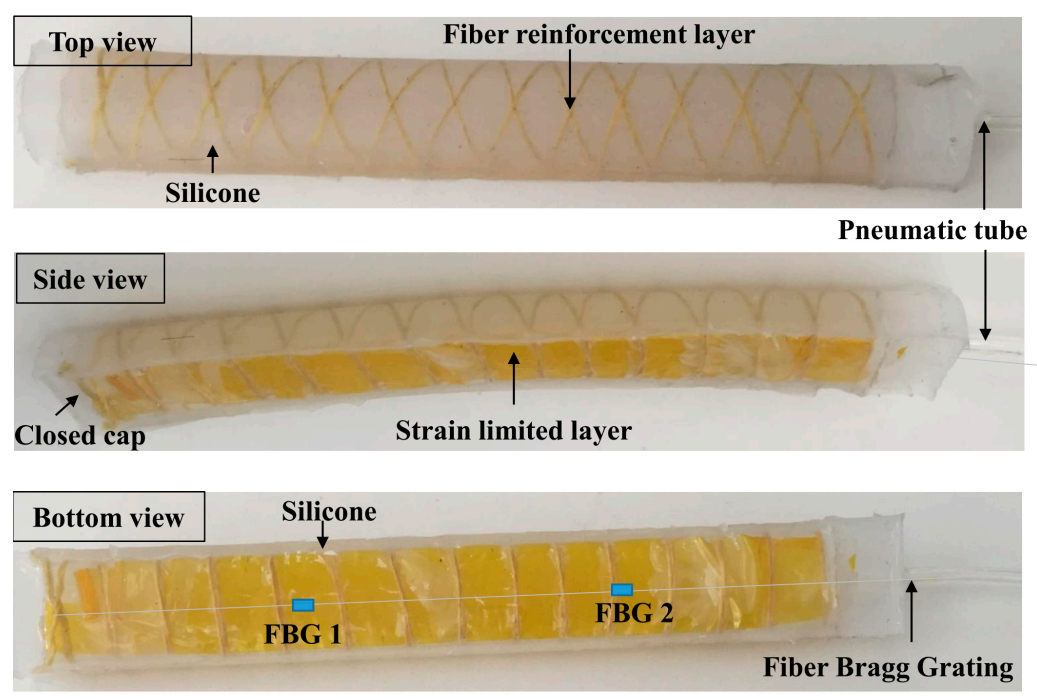

Figure 4. 3D printed mold of the soft actuator.

\section{Principle of Sensing and Measurement of Embedded FBGs}

According to the sensing principle of embedded FBG sensor, the relationship between the reflected wavelength and the grating period of FBG sensor can be expressed as [5,10]:

$$
\lambda_{B}=2 n_{e f f} \cdot \Lambda
$$

where $n_{\text {eff }}$ represents the effective index of the fiber's refractive, $\Lambda$ represents the grating period.

The grating period of FBG sensor will be changed due to the changes in the strain of the grating and the temperature, and then the reflected wavelength will be changed. The relation between the strain, temperature change, and the wavelength shift is governed by $[6,7]$ :

$$
\Delta \lambda_{B} / \lambda_{B}=\left(1-P_{\varepsilon}\right) \cdot \varepsilon+\left(\alpha_{T}+\xi\right) \cdot \Delta T
$$

where $\Delta \lambda_{B}$ is the change of wavelength, $\varepsilon$ is the strain of soft actuator, $P_{\varepsilon}$ is a constant value calculated from the photo-elastic coefficient of the fiber material, $\alpha_{T}$ is the grating thermal coefficient of expansion, $\xi$ is the grating thermo-optical coefficient, $\Delta T$ is the change of temperature.

Considering the soft surgical actuators are commonly used at room temperature with minor variation, so the influence of the temperature is ignored in this paper. Then Equation (11) can be written as Equation (12). The strain of soft actuator can be obtained from the wavelength shift of the FBGs and the photo elastic coefficient of the optical fiber [19-22];

$$
\Delta \lambda_{B} / \lambda_{B}=\left(1-P_{\varepsilon}\right) \cdot \varepsilon
$$

In this study, two FBG sensors were integrated into the polyimide strain limited layer which was embedded into the soft actuator, the relative position and bending deformation of the FBG sensors in a microstructure of the soft actuator under an actuating force is schematically shown in Figure 5. The length and thickness of the microstructure is assumed as $L$ and $h$, as Figure 5 a shows.

The pure bending model is an extensively used model representing the bending moment [10]. In this paper, an optical fiber with two FBG sensors embedded in an offset position from the neutral line, which is depicted in Figure $5 b$, is the dashed line E-F. The distance from the FBG sensor to the outer arc of the soft silicone sheet is $h_{1}$, and the distance from the FBG sensor to the inner arc of the soft silicone sensing sheet is $h_{2}$. When the optical fiber sensor is bending, as shown in Figure $5 \mathbf{b}$, 
the relationship between the bending radius, bending angle and the length of the neutral line can be expressed as:

$$
L_{E F}=R \cdot \theta
$$

where $L_{E F}$ represents the constant value of the length of the polyimide tape in neutral line, $R$ represents the radius of the neutral line, $\theta$ represents the central angle of the neutral line.

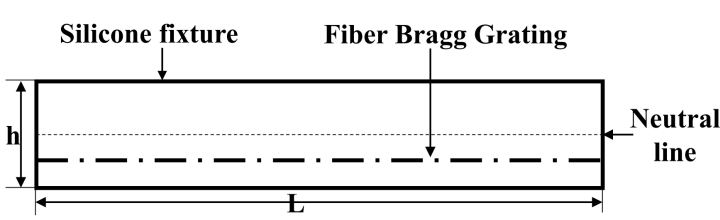

(a)

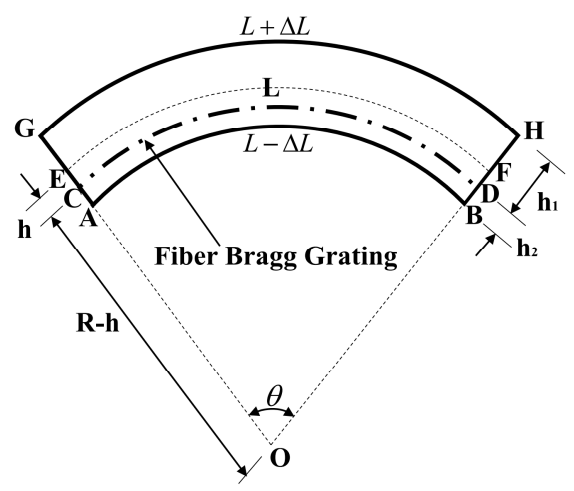

(b)

Figure 5. Bending mechanism of the FBG-based optical fiber sensor. (a) Free-state without bending. (b) Bending situation.

As the pneumatic actuating forces are applied on the soft actuator, the inner side of the micro structure is pressed, shortening the length, and the outer side of the microstructure is pulled and extending the length. Under the bending condition, the length variation of both inner and outer side is assumed as the same, and the length of the neutral layer does not change. In this paper, the FBG sensors and the polyimide sheet are considered as undergoing the same bending, due to the optical fiber being very thin and glued together with the polyimide tape. The FBG sensor is compressed during bending, so the offset location $(x)$ of the FBG sensor leads to a slight change in the bending radius and is expressed as $R-x$, the length of FBG $\left(L_{A B}\right)$ under an applied curvature can be written as:

$$
\begin{aligned}
L-\Delta L=L_{A B} & =(R-x) \cdot \theta=\left(R-\left(h_{1}+h_{2} / 2\right)\right) \cdot \theta \\
L+\Delta L=L_{G H} & =(R+x) \cdot \theta=\left(R+\left(h_{1}+h_{2}\right) / 2\right) \cdot \theta \\
x & =h_{1}-\left(h_{1}+h_{2}\right) / 2
\end{aligned}
$$

where $x$ represents the distance from the FBG to the neutral line. From Equations (14) and (15), the change of FBG length caused from the bending was obtained, and the grating period is changed proportionally to the bending angle $\theta$, the bending curvature of the microstructure can be written as [10]:

$$
C=\frac{1}{R}=\frac{2}{h} \frac{\Delta L}{L}=\frac{2}{h} \varepsilon=\frac{\Delta \lambda_{B}}{\lambda_{B}\left(1-R_{\varepsilon}\right) \cdot h}
$$

where $C$ is the bending curvature of the microstructure, $R$ represents the curvature radius, $\Delta L$ represents the length variation of both the inner and outer side of the microstructure, $\varepsilon$ is the strain of the microstructure. For any FBG sensors, $\lambda_{B}, R_{\varepsilon}, h$ are the constant, so the bending curvature and wavelength shift has a linear relationship, and this characteristic could realize the 3D shape reconstruction of the microstructure.

The shape reconstruction of the soft actuator includes curvature acquisition, curvature continuum, curve reconstruction and shape reconstruction, etc. Due to the curvature data being discrete and obtained from the FBG sensors, the curvature is serialized continuously through the linear interpolation and the fitted algorithm; then the geometrical structure of the soft actuator can be reconstructed. By using the idea of differential calculus, a line with fixed length can be divided into several uniform 
sections, and the length of these sections expressed as $L$, namely $O_{1} O_{2}=O_{2} O_{3}=L$, and then become some arcs with the length of $L$ when the microstructure is bending, and the center angle of the circular arc is determined by the length $L$ and the curvature $C$. Assuming that the coordinate of $O_{2}$ is $(0,0)$, the coordinate of $O_{3}$ can be calculated as $\left(\sin \theta_{2} / C_{2},\left(1-\cos \theta_{2} / C_{2}\right)\right)$, from the length $L$ and curvature $C$, the $\theta_{2}$ is equal to $L^{*} C_{2}$; thus this algorithm can be applied for 3D shape reconstruction of the soft actuator [23-29].

The sensing surface formed by some sensing points can be reconstructed based on the motion coordinate system, as illustrated in Figure 6. Assuming that the original curved surface along the direction of $\left(O_{1}, Z_{1}\right)$, and could bend from the direction of curves $O_{1}, O_{2}, O_{3}$, the point $O_{2}$ could be obtained from translation and rotation of the coordinate $\mathrm{O}_{2}{ }^{1}$. In the fixed length of arc, the coordinate system is set up in the tangent direction of the curve with points $\mathrm{O}_{2}$ and $\mathrm{O}_{3}$ respectively, and for the coordinate $X_{1}-Z_{1}$ and $X_{2}-Z_{2}$, the direction and size of vector $O_{1} O_{2}$ is invariable. The rotation angle $\theta_{1}$ can be calculated from the curvature $C_{1}$ (the coordinate of $O_{1}$ ), and the rotation angle $\theta_{2}$ can be calculated from the curvature $C_{2}$ (the coordinate of $O_{2}$ ).

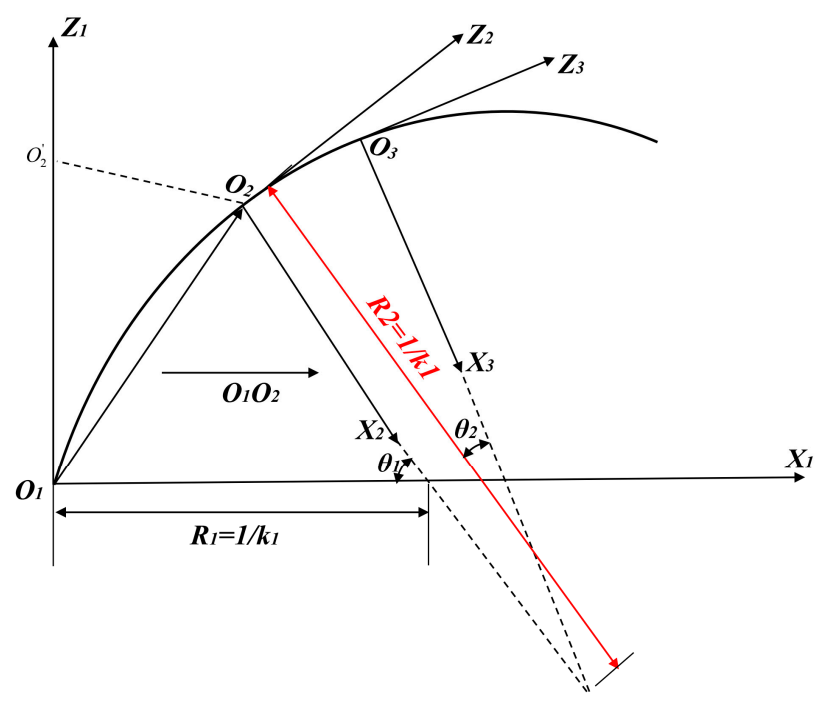

Figure 6. The algorithm of 3D shape reconstruction.

The relationship between each coordinate can be established by the rotation matrix, the ratio angle of the whole plane is fitted through the change of the curvature; thus the reconstruction and visualization of the soft actuator can be realized, and the rotation matrix can be expressed as:

$$
\left[\begin{array}{l}
X_{2} \\
Z_{2}
\end{array}\right]=\left[\begin{array}{l}
X_{1} \\
Z_{1}
\end{array}\right]=\left[\begin{array}{c}
\cos \theta_{1}-\sin \theta_{1} \\
\sin \theta_{1}-\cos \theta_{1}
\end{array}\right]
$$

Consequently, according to the curvature obtained from each sensing point, the 3D shape of the soft actuator is well reconstructed, and the real-time interaction surface with the soft actuator can be realized by using the multi-resolution mesh modeling tool. As introduced in [30-32], the 3D shape of the soft actuator can be obtained from the managing meshes with different refinement levels [30-32].

\section{Experiment Testing and Discussions}

\subsection{Pressure and Bending Angle}

In order to control the bending angle of the soft actuator, some experiments were carried out to evaluate its relationship between input air pressure and bending angle. The input pressure was controlled by a pneumatic valve in the experiment, and some experimental results were obtained 
with the pressure of $0,10,20,50,80 \mathrm{kPa}$, and each experiment was repeated ten times. An AIRTAC VALVE (GPR30008L, AirTac, Taiwan, 2016) model was used to input pressure in the experiment; the received optical signals are translated into digital signals and extracted by the computer. A LabVIEW (LabVIEW 2014) program is developed to store the data and reconstruct the bending shape of the soft actuator [18-20]. In some robotic applications, the range of the soft surgical manipulation motion is more important than its actuation velocity, so the velocity of actuation is seldom considered. Consequently, for the sake of avoiding some dynamic oscillations, the bending motion of the soft actuator generated at a slow pressure rate of $0.2 \mathrm{~Hz}$. Figure 7 shows some snapshots of the bending movement of the soft actuator, and an improved track learning detection algorithm was applied to recording the motion trajectory of the soft actuator.

The average bending angle of the soft actuator was calculated from the same input pressure, as depicted in Figure 8. The theoretical and experimental results of the bending trajectories of the soft actuator are presents in Figure 9.

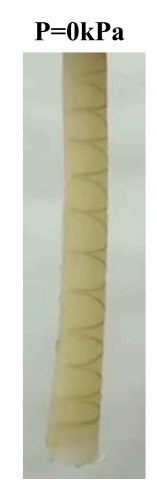

(a)

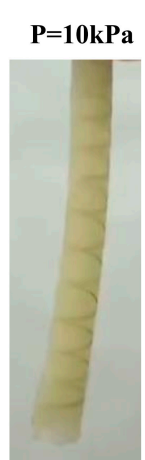

(b)

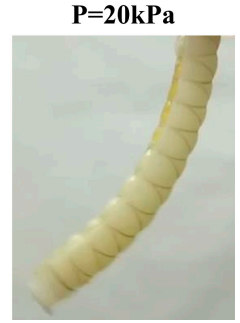

(c)

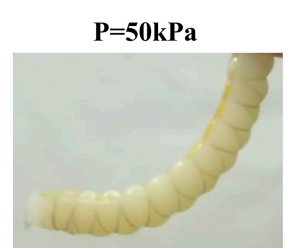

(d)

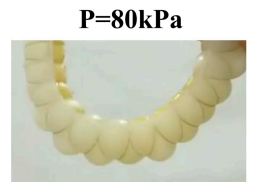

(e)

Figure 7. Experiment view of the actuator with different input pressure. (a) Bending state. (b) State 2. (c) State 3. (d) State 4. (e) State 5.

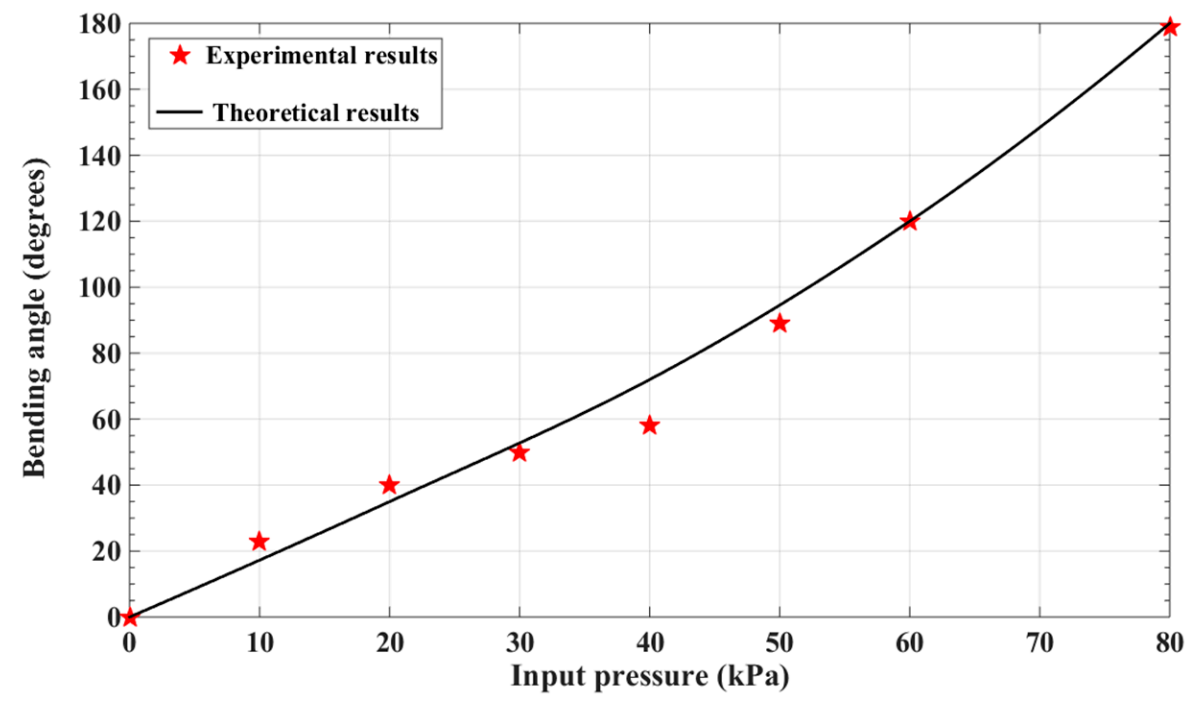

Figure 8. Input pressure against bending angle results of soft actuator. 


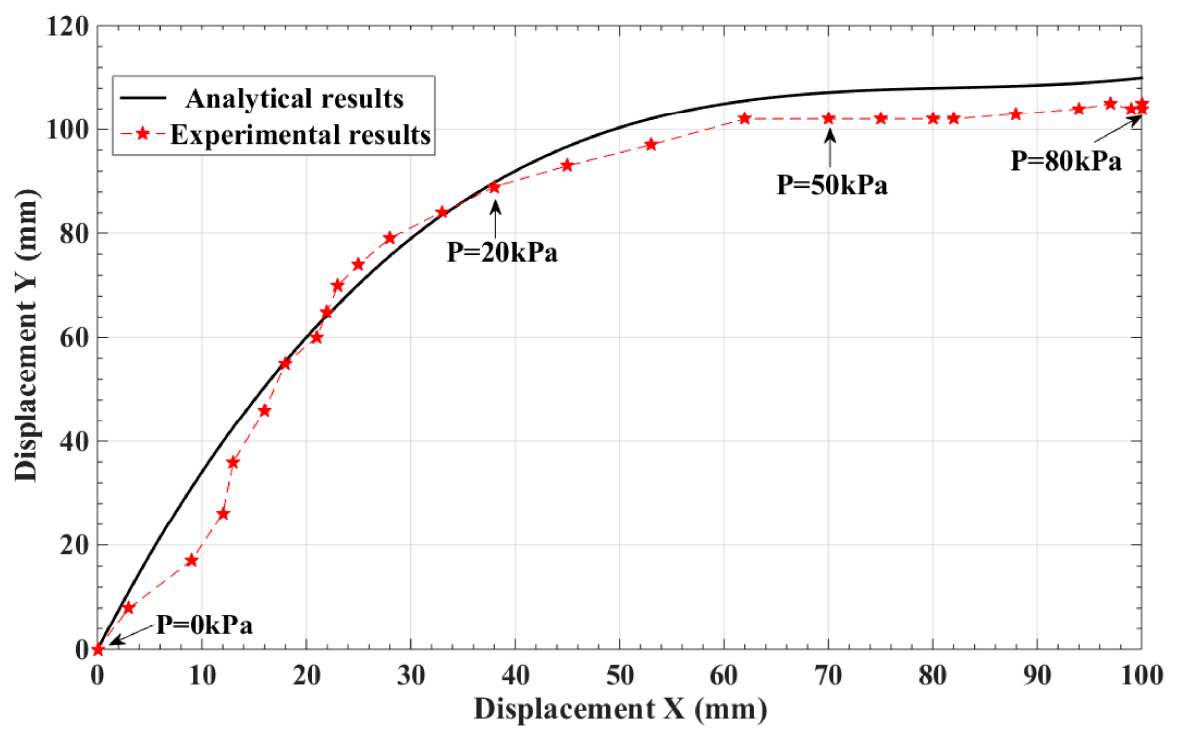

Figure 9. Motion trajectory of the actuator with different input pressure.

As depicted in Figure 8, due to the radial bulging effects of the base layer and extension of the soft silicone actuator, there exists some minor error between the analytical and experiment results, and a repeatable nonlinear pattern of results possibly caused by the gravity of the actuators, and the center of gravity of soft actuator is changing in different bending state. As depicted in Figure 9, although the actual motion trajectory of the soft actuator is not smooth and has some deviations, there was only a slight deviation between the theoretical and experimental results, so the design validity and effectiveness of the soft actuator was demonstrated.

\subsection{Shape Reconstruction of the Soft Actuator}

In order to demonstrate the effectiveness of the shape reconstruction method proposed in this paper, some bending experiments of the soft actuator based on embedded FBGs were carried out. The experimental setup is illustrated in Figure 10. In the experiment, the original center wavelengths of two FBGs (FBG1, FBG2) are $1544 \mathrm{~nm}$ and $1546 \mathrm{~nm}$ respectively. The peak reflectivity of the FBGs are $70 \%$ and the response time of the FBGs is $70 \mathrm{~ms}$. Two FBG sensors are located in the intermediate axis of the soft actuator, and their relative positions as illustrated in Figure 4, Section 2.

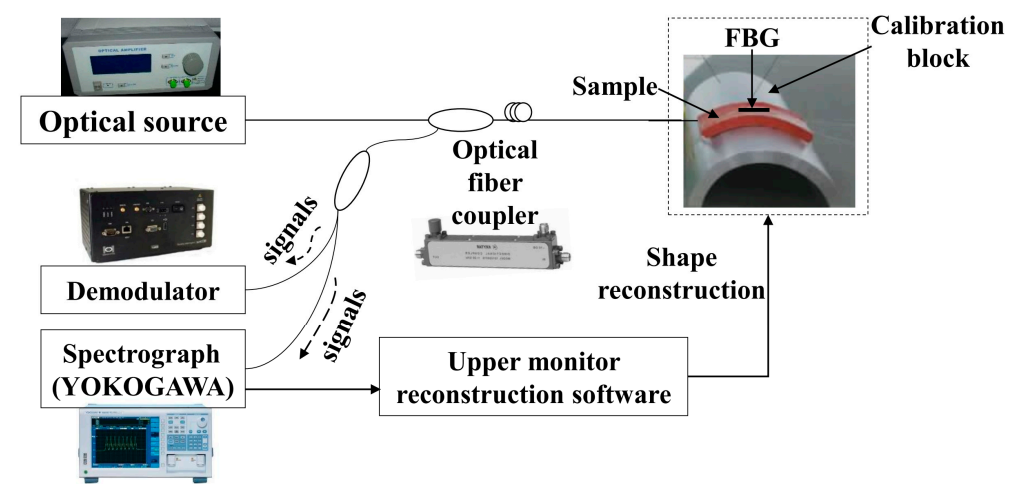

Figure 10. Experimental setup of the soft actuator with embedded FBGs.

As the bending curvature of the soft actuator varies with $0 \mathrm{~m}^{-1}, 5 \mathrm{~m}^{-1}, 10 \mathrm{~m}^{-1}, 15 \mathrm{~m}^{-1}, 20 \mathrm{~m}^{-1}$ and $25 \mathrm{~m}^{-1}$, the wavelength and power of the FBG sensors are not the same as the original values. Since the grating characteristics of FBG sensors are varied from the strain loads of polyimide tape as it 
bends, the power peaks of the FBGs can be identified accurately. As depicted in Figure 11, the power peaks of the two FBGs were around $1544.498 \mathrm{~nm}$ and $1546.611 \mathrm{~nm}$ at bending state 1 , the curvature of the polyimide skin was increased when the soft actuator bent from state 1 to state 5 , and the power peaks of the two FBGs was $1545.956 \mathrm{~nm}$ and $1547.541 \mathrm{~nm}$ at bending state 5. From Figure 11, it also indicates that the peak wavelength gradually shifts in the direction of a longer wavelength as the bottom surface curvature of the soft actuator increases from $0 \mathrm{~m}^{-1}$ to $25 \mathrm{~m}^{-1}$, and the shift value of the two FBG sensors is about $1.458 \mathrm{~nm}$ and $0.93 \mathrm{~nm}$, respectively. Consequently, the responses of the two FBGs are sensitive and instantaneous record the variation in power. The dynamic shape deformation of the soft actuator can be measured in real time, and the FBG sensors with different wavelengths can be distributed along a single optical fiber for multi-point shape sensing.

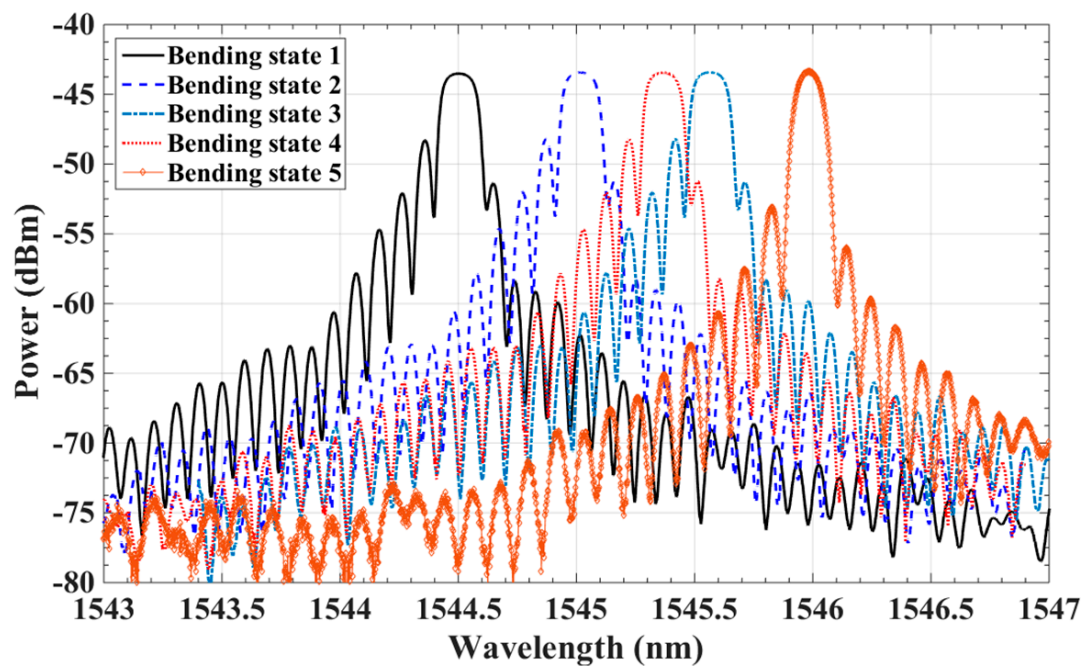

(a)

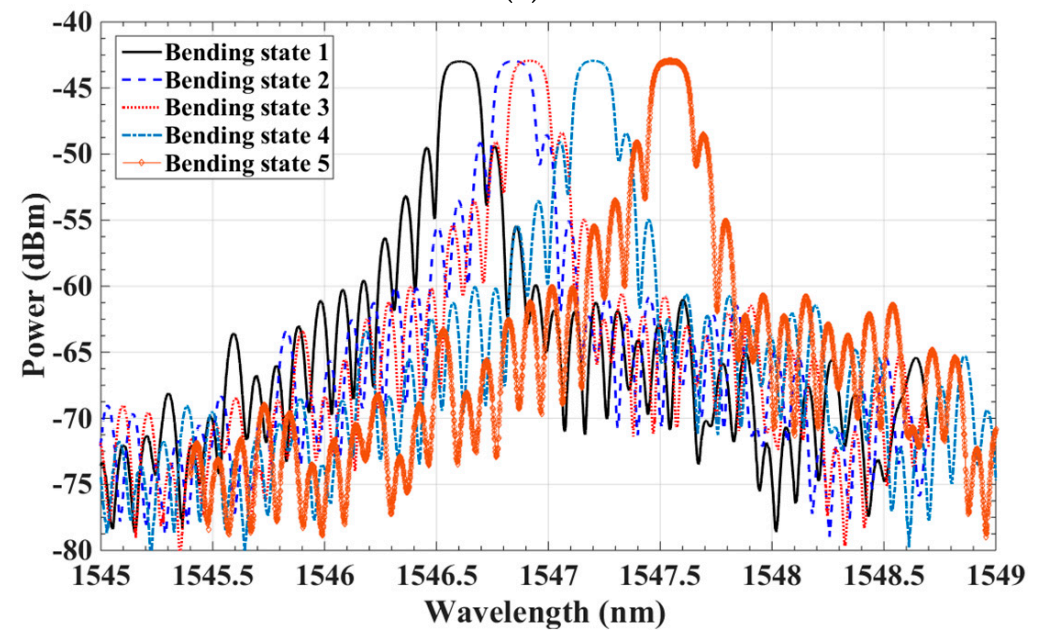

(b)

Figure 11. The wavelength shift of two FBG sensors at different bending states. (a) FBG 1. (b) FBG 2.

When the curvature of the FBGs increases from $0 \mathrm{~m}^{-1}$ to $25 \mathrm{~m}^{-1}$, the relationship of the wavelength shift and the curvatures of the soft actuator are illustrated in Figure 12. All measurements have respective average values which were taken from six independent experiments. The bending curvature of the soft actuator at different bending states obtained from the wavelength shift of the FBG sensors, the interpolation algorithm, and the wavelength shift of the FBG sensors were different due to the bending produced strain loads at the different sensing positions. The results showed that the wavelength shift of the FBG sensors increased nonlinearly with the increasing bend in the curvature of the soft actuator. That is because the soft silicone material is extensible and its thickness is variable as 
it sustains different driving loads and contact forces at different bending states. The thickness of the microstructure $h$ at different sensing points varied and the strain loads induced by the deformation of the soft silicone actuator were different at these sensing points. According to Equation (17), when the bending curvature of the soft actuator increases from $0 \mathrm{~m}^{-1}$ to $25 \mathrm{~m}^{-1}$, the wavelength shift and the bending curvature presents a nonlinear variation trend. The wavelength shift of the FBG sensors can be calculated by using the method of polynomial curve fitting, and the calibration equations can also be used to test the bending curvatures of the soft actuator.

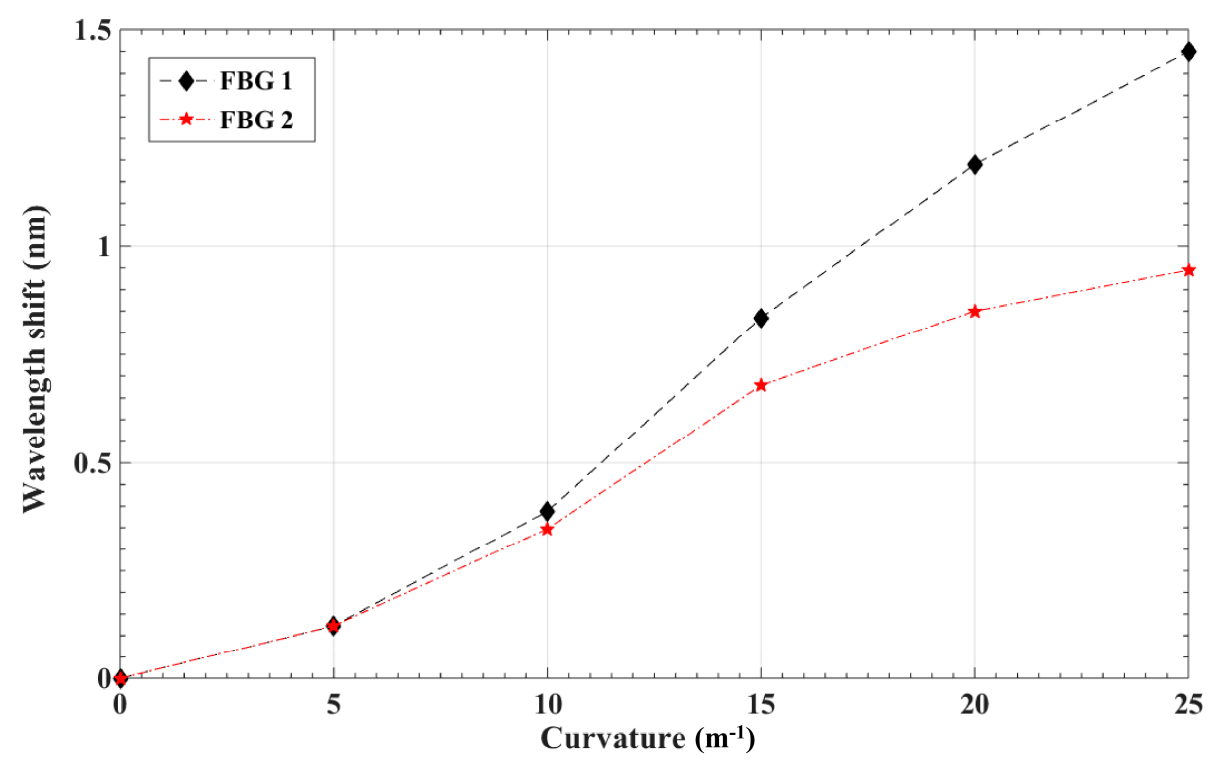

Figure 12. The wavelength shifts of two FBG sensors under different curvatures.

For the 3D shape reconstruction of the soft actuator at different bending states, the bending curvatures of the two FBG sensors were calculated from the wavelength shift. According to the data obtained from each sensing point, the 3D shape of the soft actuator is reconstructed through the interpolation and curve fitting functions. The 3D shapes reconstruction results of the soft actuator with different bending state numbered from 1 to 5 are presents in Figure 13. From the figure, the shape of the actuator is well reconstructed, and the spatial resolution determined by the density of FBG sensors in the sensing point is about $30 \mathrm{~mm}$. Consequently, the feasibility and effectiveness of the 3D shape reconstruction method of the soft actuator based on embedded FBG sensors are demonstrated.

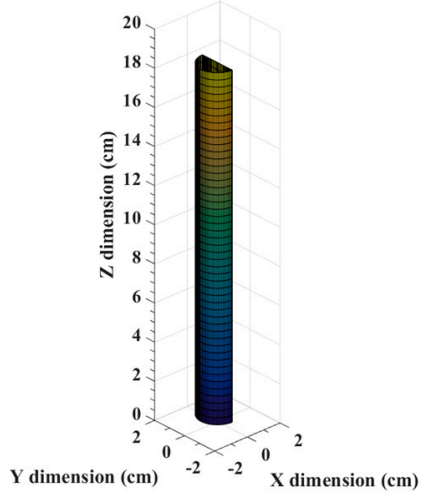

(a)

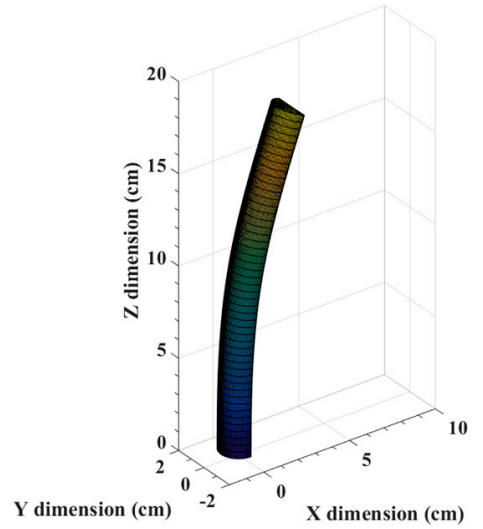

(b)

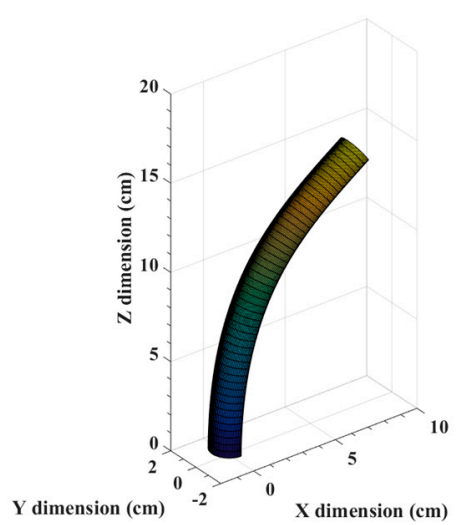

(c)

Figure 13. Cont. 


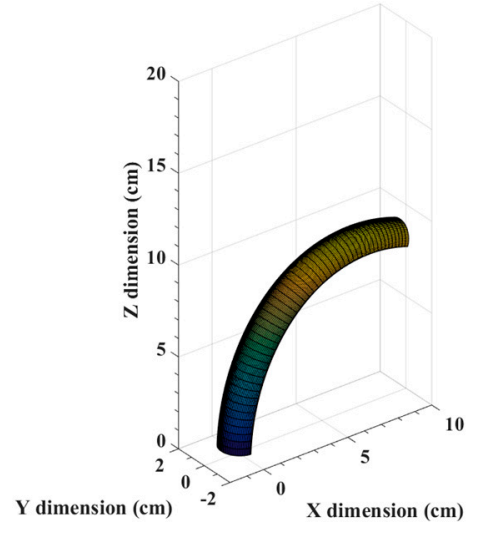

(d)

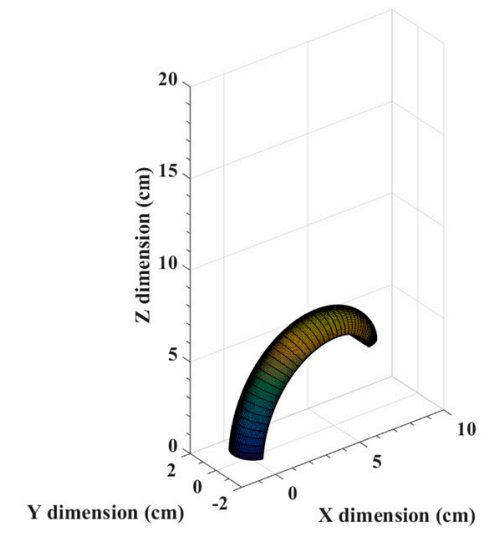

(e)

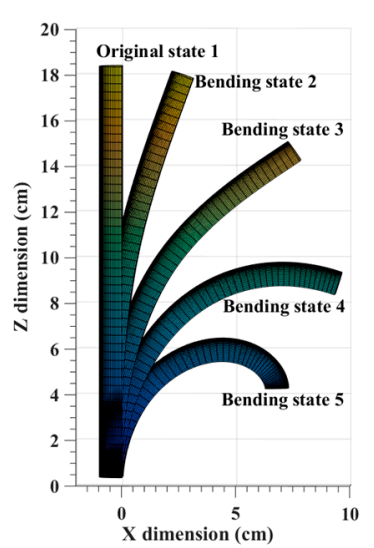

(f)

Figure 13. Shape reconstruction of the soft actuator at different bending state. (a) Original state 1. (b) Bending state 2. (c) Bending state 3. (d) Bending state 4. (e) Bending state 5. (f) Bending state 1 to 5.

\section{Conclusions}

To implement real-time shape monitoring of soft surgical actuator, this paper proposes a 3D shape reconstruction method of a soft actuator that has an embedded optical fiber with two FBG sensors. First, the design and measurement principle of the soft actuator based on the FBG sensors are analyzed and described. An optical fiber with two FBG sensors are embedded into a soft actuator by a polyimide tape and encapsulated by the silicone layer. Second, the relation curve of the wavelength shifts of the FBG sensors and bending curvatures of the soft actuator are obtained, and which can be used to measure the bending curvatures of the soft actuator at different bending state. Then, the peaks of the reflected wavelengths and the variation of the reflection spectrums are acquired, the sensitive and instantaneous response of FBGs sensors are reflected. Finally, according to the data from the FBG sensing points, the 3D shape of the soft actuator is well reconstructed through the interpolation and curve fitting functions. This well reconstructed 3D shape of the soft actuator demonstrates the effectiveness of the shape reconstruction method that is proposed in this paper, as well as the potential and increased applications for real-time shape monitoring in the field of soft robotics and flexible biosensor monitoring. In the future, the dynamic behavior and closed-loop controlling of the soft actuator will be investigated, and the application of soft actuator in some surgical operations will be extended.

Author Contributions: L.Z. and G.S. conceived and designed the experiments; M.Y. performed the experiments; Y.H. analyzed the data and wrote the paper; M.D. contributed reagents/materials/analysis tools.

Acknowledgments: This work is supported by the Beijing Information Science and Technology University funded project (No. 1825008). This research project was also supported by the Changjiang Scholars and Innovative Research Team in University (No. IRT_16R07), Importation and Development of High-Caliber Talents Project of Beijing Municipal Institutions (No. IDHT20170510), China Postdoctoral Science Foundation funded project (2018M631290).

Conflicts of Interest: The authors declare no conflict of interest.

\section{References}

1. Ranzani, T.; Gerboni, G.; Cianchetti, M.; Menciassia, A. A bioinspired soft manipulator for minimally invasive surgery. Bioinspir. Biomim. 2015, 10, 035008. [CrossRef] [PubMed]

2. Zolfagharian, A.; Kouzani, A.Z.; Khoo, S.Y.; Moghadam, A.A.A.; Gibson, L.; Kaynak, A. Evolution of 3D printed soft actuators. Sens. Actuators A Phys. 2016, 250, 258-272. [CrossRef]

3. Luo, M.; Skorina, E.H.; Tao, W.; Chen, F.C.; Ozel, S.; Sun, Y.N.; Onal, C.D. Toward modular soft robotics: Proprioceptive curvature sensing and sliding mode control of soft bidirectional bending modules. Soft Robot. 2017, 4, 1-9. [CrossRef] [PubMed] 
4. Polygerinos, P.; Wang, Z.; Overvelde, J.T.B.; Galloway, K.C.; Wood, R.J.; Bertoldi, K.; Walsh, C.J. Modeling of soft fiber-reinforced bending actuators. IEEE Trans. Robot. 2015, 31, 778-789. [CrossRef]

5. Sun, G.; Li, H.; Dong, M.; Lou, X.; Zhu, L. Optical fiber shape sensing of polyimide skin for a flexible morphing wing. Appl. Opt. 2018, 56, 9325. [CrossRef] [PubMed]

6. Nguyen, T.D.; Han, H.S.; Shin, H.Y.; Nguyen, C.T.; Phung, H.; Hoang, H.V.; Choi, H.R. Highly sensitive flexible proximity tactile array sensor by using carbon micro coils. Sens. Actuators A Phys. 2017, 266, 166-177. [CrossRef]

7. Ozel, S.; Keskin, N.A.; Khea, D.; Onal, C.D. A precise embedded curvature sensor module for soft-bodied robots. Sens. Actuators A Phys. 2015, 236, 349-356. [CrossRef]

8. Wang, H.S.; Zhang, R.X.; Chen, W.D.; Liang, X.W. Shape detection algorithm for soft manipulator based on Fiber Bragg Gratings. IEEE/ASME Trans. Mechatron. 2016, 21, 2977-2981. [CrossRef]

9. Payo, I.; Feliu, V.; Cortazar, O.D. Fibre Bragg grating (FBG) sensor system for highly flexible single-link robots. Sens. Actuators A Phys. 2009, 150, 24-39. [CrossRef]

10. Ge, J.; James, A.E.; Li, X.; Chen, Y.; Kwok, K.W.; Fok, M.P. Bidirectional soft silicone curvature sensor based on off-centered embedded fiber bragg grating. IEEE Photonics Technol. Lett. 2016, 28, 2237-2240. [CrossRef]

11. Kim, S.W.; Kang, W.R.; Jeong, M.S.; Lee, I.; Kwon, I.B. Deflection estimation of a wind turbine blade using FBG sensors embedded in the blade bonding line. Smart Mater. Struct. 2013, 22, 1-11. [CrossRef]

12. Silva, A.S.; Catarino, A.; Correia, M.V.; Frazão, O. Design and characterization of a wearable macro bending fibre optic sensor for human joint angle determination. Opt. Eng. 2013, 52, 992-999. [CrossRef]

13. Searle, T.C.; Althoefer, K.; Seneviratne, L.; Liu, H.B. An optical curvature sensor for flexible manipulator. In Proceedings of the 2013 IEEE International Conference on Robotics and Automation (ICRA), Karlsruhe, Germany, 6-10 May 2013; pp. 4415-4420. [CrossRef]

14. Sareh, S.; Noh, Y.; Li, M.; Ranzani, T.; Liu, H.B.; Althoefer, K. Macrobend optical sensing for pose measurement in soft robot arms. Smart Mater. Struct. 2015, 24, 125024. [CrossRef]

15. Zhao, H.; Jalving, J.; Huang, R.K.; Knepper, R.; Ruina, A.; Shepherd, R. A helping hand: Soft orthosis with integrated optical strain sensors and EMG control. IEEE Robot. Autom. Mag. 2016, 23, 55-64. [CrossRef]

16. Connolly, F.; Walsh, C.J.; Bertoldi, K. Automatic design of fiber-reinforced soft actuators for trajectory matching. Proc. Natl. Acad. Sci. USA 2017, 114, 51-56. [CrossRef] [PubMed]

17. Polygerinos, P.; Wang, Z.; Galloway, K.C. Soft robotic glove for combined assistance and at-home rehabilitation. Robot. Autonom. Syst. 2015, 73, 135-143. [CrossRef]

18. Ranzani, T.; Cianchetti, M.; Gerboni, G.; De Falco, I.; Menciassi, A. A soft modular manipulator for minimally invasive surgery: Design and characterization of a single module. IEEE Trans. Robot. 2016, 32, 187-200. [CrossRef]

19. Laschi, C.; Mazzolai, B.; Cianchetti, M. Soft robotics: Technologies and systems pushing the boundaries of robot abilities. Sci. Robot. 2016, 1, 3690. [CrossRef]

20. Zhao, Z.; Soto, M.A.; Tang, M.; Thevenaz, L. Distributed shape sensing using Brillouin scattering in multi-core fibers. Opt. Express 2016, 24, 25211-25223. [CrossRef] [PubMed]

21. Guo, J.J.; Liu, X.Y.; Jiang, N.; Yetisen, A.K.; Yuk, H.; Yang, C.X.; Khademhosseini, A.; Zhao, X.H.; Yun, S.-H. Highly stretchable, strain sensing hydrogel optical fibers. Adv. Mater. 2016, 28, 10244-10249. [CrossRef] [PubMed]

22. Qiu, Y.; Shen, L.; Hu, W. Shape rebuilding and positioning method of search and rescue robot endoscope in ruin crack. Chin. J. Sci. Instrum. 2015, 36, 2782-2789.

23. Robertson, M.A.; Paik, J. New soft robots really suck: Vacuum-powered systems empower diverse capabilities. Sci. Robot. 2017, 2, 1-11. [CrossRef]

24. Cianchetti, M.; Ranzani, T.; Gerboni, G.; Nanayakkara, T.; Althoefer, K.; Dasgupta, P.; Menciassi, A. Soft robotics technologies to address shortcomings in today's minimally invasive surgery: The stiff-flop approach. Soft Robot. 2014, 1, 122-131. [CrossRef]

25. Wang, Y.; Negahdaripour, S.; Aykin, M.D. Calibration and 3D reconstruction of underwater objects with non-single view projection model by structured light stereo imaging. Appl. Opt. 2016, 55, 6564-6575. [CrossRef] [PubMed]

26. Westbrook, P.S.; Kremp, T.; Feder, K.S.; Ko, W.; Monberg, E.M.; Wu, H.C.; Simoff, D.A.; Taunay, T.F.; Ortiz, R.M. Continuous multicore optical fiber grating arrays for distributed sensing applications. J. Lightw. Technol. 2017, 35, 1248-1252. [CrossRef] 
27. Parent, F.; Loranger, S.; Mandal, K.K.; Iezzi, V.L.; Lapointe, I.; Boisvert, J.S.; Baiad, M.D.; Kadoury, S.; Kashyap, R. Enhancement of accuracy in shape sensing of surgical needles using optical frequency domain reflectometry in optical fibers. Biomed. Opt. Express 2017, 8, 2210-2221. [CrossRef] [PubMed]

28. Waltermann, C.; Doering, A.; Kohring, M.; Angelmahr, M.; Schade, W. Cladding waveguide gratings in standard single-mode fiber for 3D shape sensing. Opt. Lett. 2015, 40, 3109-3112. [CrossRef] [PubMed]

29. Feng, D.Y.; Zhou, W.J.; Qiao, X.G.; Albert, J. Compact optical fiber 3D shape sensor based on a pair of orthogonal titled fiber bragg gratings. Sci. Rep. 2015, 17415. [CrossRef] [PubMed]

30. Song, S.; Li, Z.; Yu, H.; Ren, H. Electromagnetic positioning for tip tracking and shape sensing of flexible robots. IEEE Sens. J. 2015, 15, 4565-4575. [CrossRef]

31. Quandt, B.M.; Fan, L.J.H.; Qian, J. Vibration shape testing of space sheet structure. J. Shanghai Univ. 2000, 14, 354-358.

32. Lee, K.K.C.; Mariampillai, A.; Haque, M.; Standish, B.A.; Yang, V.X.D.; Herman, P.R. Temperature-compensated fiber-optic 3D shape sensor based on femtosecond laser direct-written Bragg grating waveguides. Opt. Express 2013, 21, 24076-24086. [CrossRef] [PubMed]

(C) 2018 by the authors. Licensee MDPI, Basel, Switzerland. This article is an open access article distributed under the terms and conditions of the Creative Commons Attribution (CC BY) license (http:/ / creativecommons.org/licenses/by/4.0/). 